\title{
Quality Improvement Methodologies - PDCA Cycle and Six Sigma in E-learning System
}

\author{
Manal Alyoubi ${ }^{1}$, Jawaher Alqahtani ${ }^{2}$, Azrilah Abd.Aziz ${ }^{3}$ \\ Information Systems Department, Faculty of Computing \& Information Technology, King Abdulaziz University, \\ Jeddah, Saudi Arabia ${ }^{1,2}$
}

$\mathrm{PhD}$, Information Systems Department, Faculty of Computing \& Information Technology, King Abdulaziz University, Jeddah, Saudi Arabia ${ }^{3}$

\begin{abstract}
With increasing demand for E-learning, there is growing concern about the ability to ensure its quality. This paper identifies the e-learning system quality issues, which are related to the user, teacher, course, design, technology, and environment. Then the paper discusses how to implement the Total Quality Management (TQM) in e-learning system to achieve the most advantages from it.
\end{abstract}

Keywords: Total quality management, E-learning, PDCA Cycle, Six Sigma.

\section{INTRODUCTION}

E-learning is more than offering on-line courses. It changed to utilizing innovation to convey part or the majority of a course autonomous of perpetual time and place. Also, it describes as "the use of new multimedia technologies and the Internet to increase learning quality by easing access to facilities and services as well as distant exchanges and collaboration" [1]. E-learning alludes to the utilization of information and communication technologies to enable the access to online learning/teaching resources.

'E-learning' comes in different terms due to the diversity of the perspectives, for instance, online-distance learning, hybrid learning and distributed learning [1].

In the last decade, big investments have directed toward information and communications technology (ICT) in education and learning. Despite that, there are some example of failure project, for instance, UK eUniversity and Global University Alliance[5]. For that, different Institutions have developed some quality models and benchmarks to use them. Total Quality Management (TQM) has introduced, to overcome such failure and the quality issues in elearning [15].

In this paper, we review first the advantages and disadvantages of e-learning system. Then we discuss the concept of TQM and the past, present, and future of the quality management. After that, we highlight the principles of TQM implementation, the quality issues in the e-learning system. Next, present the implementation of TQM in e-learning system and the challenges that are faced it.

\section{ADVANTAGES OF E-LEARNING SYSTEM}

A few reviews give preferred standpoint of e-learning as its capacity to concentrate on the requirements of learners.

Some advantages of e-Learning:

1. The issues of time and place are not a concern. Each Learner can pick the location and time that suits him/her.

2. By using the e-learning, we ensure the viability of information, with the simplest way to access it.

3. A lot of people may be afraid of showing their thought and ideas in front of others (Social anxiety disorder).But through e-learning, that obstacle disappears. The learners can interact by the utilization of electronic forums.

4. The learner in e-learning does not need travel and mobility to study. It is practical as it eliminates all obstacles in a traditional education.

5. E-learning support the individual learner's variance. The learners may like to concentrate on particular sections of the course, while the rest may be interested in auditing the whole course.

6. E-learning Solve the problem of lack of scholarly staff, including educators and additionally facilitators, lab specialists and so forth.

7. It supports self-paced learning. For instance, the non-concurrent way allows each understudy to learn at his or her pace and speed whether moderate or fast. 


\section{III.DISADVANTAGES OF E-LEARNING SYSTEM}

The odds of e-learning that have been given by studies incorporate the accompanying [1]:

1. As it is a way for instruction makes the learners experience consideration, remoteness, and additionally absence of collaboration or connection. It subsequently requires an exceptionally stable motivation as well as abilities with to the administration of time so as to decrease such impacts.

2. Concerning elucidations, offer of clarifications, and understandings, the e-learning strategy may be less compelling that the conventional technique for learning. The learning procedure is significantly less demanding with the utilization of the up close and personal experience with the educators or instructors.

3. With regards to change in relational abilities of learners, e-learning as a technique might have a negative impact.

4. E-learning may likewise most likely be deluded to theft and written falsification, inclined by lacking determination aptitudes.

5. E-learning may degenerate the socialization foundations' part and even more the teachers' role as the administrators of the instruction system.

6. Additionally, not all fields can utilize the e-learning method in instruction. The logical fields that incorporate common sense cannot be appropriate concentrates through e-learning. Examines have contended that e-learning is more suitable in sociology and humanities than the logical fields, for example, restorative science, and drug store, where there is the need to create viable abilities.

7. E-learning may likewise prompt clog or substantial utilization of a few sites. That may bring about unexpected costs both in time and cash drawbacks.

\section{IV.PAST, PRESENT AND FUTURE OF QUALITY MANAGEMENT}

Several shifts have been happening to the quality management concept over the last decades. The inspection was the first method used in looking for defects. It is done in different ways, but in general, it is based on comparing the product/service with certain specifications to isolate the bad ones. It is subject to inspector's opinion, condition and waste so many efforts, time and money. Later on, the concept of total quality management appeared as a result of quality gurus' efforts. The first quality guru was Edward Deming who introduced the management method, consisted of 14 points [2].

Joseph M Juran also believed in quality planning. He has many contributions such as the Trilogy Quality Planning (quality planning, quality control, and quality improvement) and Praetor principle [13]. Philip Crosby focused on the output by reducing defects in the performance. And setting the standards that should not only measures the imbalance but also estimates the total cost [6]. This field is rich with a lot of contributions made by other quality gurus. But lately, different initiatives based on total quality management appeared like Six Sigma and Lean Sigma [3].

\section{TQM CONCEPT}

Total quality management strategies have been applied widely. These strategies include process improvement in a management system along with the social and technical system. TQM is a way of managing the business, based on applying continuous improvement on every process in the organization. The two functions that form TQM are total quality control and quality management. Total quality control is about ensuring customer and employee satisfaction and the quality of product. While coordinate and direct an organization toward continuous improvement in all processes in quality management [7].

\section{VI.PRINCIPLES OF TQM IMPLEMENTATION}

Behind any successful TQM implementation, basic principles must be ensured [3]:

1. Quality is every employee's issue. Enabling the only managers to control the quality was a concept gone with the times. Now, every worker at the organization must be involved in the quality achievement processes, as he can stop the production when he detects any defects therein.

2. Customer focus. Keeping the existing customers and getting new ones are not an easy task. But that can be achieved by understanding their target; therefore they can provide them beyond their expectation.

3. Quality must be at every development stage. Having a thorough understanding of production process with applying quality, will stop defects from happening.

4. Top-down organizational commitment. Quality culture should prevail from the entire organization; otherwise, it will fail.

5. Continuous training. Continuous improvement in processes requires new skills and knowledge. For that fact, training becomes a critical factor.

6. Slogans alone don't give quality. Slogans become meaningless if it not accompanied by an action. 
7. Quality is long term implementation. TQM process needs performance measurement to ensure the efficient implementation which takes years.

8. With combined teams' effort, the quality can be achieved.

9. Used TQM tools efficiently. There are different tools can be used, for instance, benchmarks, management seven tools, and six sigma.

10. Continuous improvement. The continuous improvement in every aspect of the production process will raise the productivity and fulfill customer's wants, which eventually will ensure organization's place in the business.

\section{E-LEARNING SYSTEM QUALITY ISSUES}

Multidisciplinary, considered as one of the most standing characteristics of e-learning system. Many research fields must be involved to have a complete picture of such a system, for instance, computer science, information system, psychology, and education [12]. For that there are some qualities issues must be taken into account before applying any TQM methodology, which can be classified into six aspects [11]:

1. User aspect: Learner's ability to use technology in education. The computer is the primary element in this process, where the teacher provides the material through it; even the communication between the teacher and the student happens through the computer network. So we can conclude that: The positive acceptance of the use of computers increases the effectiveness of e-learning and vice versa. This dimension also includes: how to make the learning process enjoyable, attractive and easy for the learner.

2. Teacher aspect: The teacher should respond promptly to student' questions. Also, it includes the extent to which the teacher accepts the use of technology in the educational process.

3. Course aspect: The courses quality depends on its flexibility in providing the material in the style and time that suits the learner.

4. Design aspect: The design of the system should be easy-to-use, user-friendly, secure and stable.

5. Technology aspect: The IT devices play an essential role in delivering the material in a proper way for instance blackboards, earphones, and microphones.

6. Environmental aspect: useful assessment tools and methods increase customer satisfaction.

\section{IMPLEMENTATION OF TQM IN E-LEARNING SYSTEM}

\section{A. PDCA Cycle}

Every organization has different critical success factors (CSF), which mean, TQM model will not be the same for all. Afterwards, it has been proven that all TQM models have common assumptions and tools that can be used widely [5]. In e-learning system, TQM can help to ensure reliability, scalability and for certain delivers education with high value. E-learning can achieve its benefits to both user and the organization through implementing the PDCA (Plan-Do-CheckAct) cycle, one of the Edward Deming's methodologies to continuous improvement as shown in figure 1 [9].

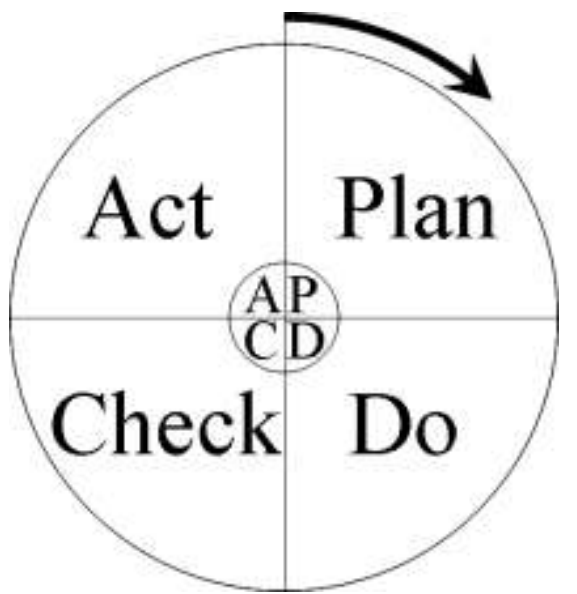

Fig.1.PDCA Cycle

PDCA is four basic sequential phases of continuous improvement, which must follow to solve the problem. The four steps that represent the method are shown below [9]:

Plan: At this stage, the causes of the problem or the task that need to be accomplished are identified. Then put a plan for it, which is done through present everybody' ideas and proposals, supported by some tools such as a Pareto principle or fishbone diagram. 
Do: Here the necessary changes are carried out gradually, to avoid confusion and disruption of routine activities. Also at this stage, staff training is provided to ensure the excellent work.

Check: When the process reaches this stage, it must check if changes have achieved the desired results or not. By make comparisons, assess the difference between the expectation and real achievement, and determine the gap between the required results and the actual results. Finally, provide the assessment to decision-makers.

Act: At this stage, adopt changes if the desired results are achieved and implemented at a greater level. Or re-plan for the next PDCA cycle.

\section{B. Six Sigma}

Having subscribed to the ISO 9001 standard and the SEI CMM, it was a short stride for TIS to research Six Sigma is an administration reasoning initially created by the Motorola association. It sets to significant degree intense targets for the accumulation of information and the examination of results. Its objectives are to lessen deserts in items and administrations beyond what many would consider possible. There are three key components to achieving "quality": customer, processes, and workers. Client characterizes quality, and expect-in addition to other things - execution, reliability, competitive costs, on-time delivery, service, clear and rectify exchange processing[8].Six sigma can be classified in light of benefits of the business and knowledge of the solution/improvement[4].

Six Sigma, as shown in figure 2 [8] is a profoundly restrained process that helps associations to concentrate on creating and conveying close flawless items and services. The word "Sigma" is a factual term that measures how far a given procedure veers off from "perfection." The focal thought behind Six Sigma is that, if you can quantify what number of "deformities" you have a procedure, you can exercise how to dispense with them and get as near "zero defects "as conceivable". A procedure must deliver close to 3.4 deformities for each million opportunities, to accomplish Six Sigma quality". An "opportunity" characterizes as any shot for "non-conformance "that is, not meeting the required details [8].

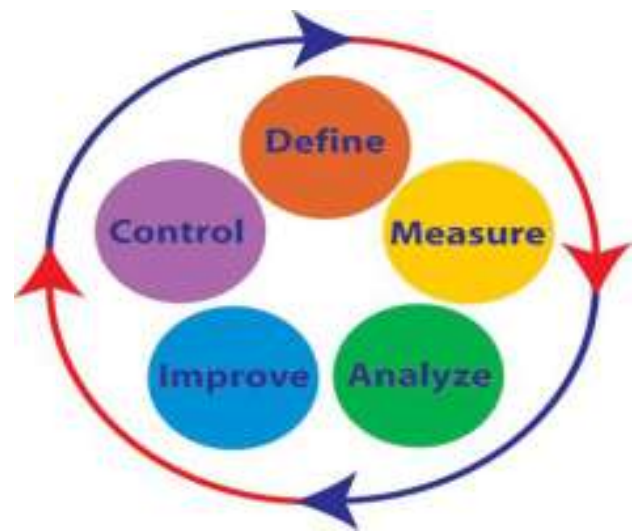

Fig.2.Six Sigma

The phases in Six Sigma [8]:

1. The "define "phase: characterizing venture objectives and limits and distinguishing issues that should be routed to accomplish the higher "sigma level".

2. The "measure "phase: gathering data about the present circumstance to acquire gauge information on current process execution and distinguish issue ranges.

3. The "analyze" phase: recognizing the crucial reasons for quality problems, using fitting information examination apparatuses.

4. The "improve" phase: actualizing arrangements that address the issues that recognized amid the past stage.

5. The "control" phase: assessing and checking the aftereffects of the past stage.

Defenders of Six Sigma keep up that six-sigma technique powers and filled by a three-stage cycle:

1. Evolving conduct.

2. Authoritative culture change.

3. Encounter the outcomes.

\section{IX.CHALLENGES OF TQM IN E-LEARNING SYSTEM}

Although systems and institutions differ, the success of using the TQM principle lies in implementation rather than only planning, and that applies to e-learning systems. In general, there are some challenges faces implementing TQM in 
e-learning systems. Verify the quality of educational content to keep up with the development of different sciences and to create a great motivation to learning. Ensure that users have all the tools that necessary to gain the maximum benefits of the scientific material, whether were software or hardware. Also, use the technology that up to date. The last factor, related to an administrative role such as continuous evaluation of results and providing training because of the rapid changes that caused by TQM [14].

\section{CONCLUSION}

Even though applying the principle of TQM started in the industry first, it has proved effective in other fields such as elearning system [5] [10]. We can conclude that quality concept is strengthening leadership, teamwork, customer satisfaction, continuous improvement and increase the profit. TQM in e-learning should focus on six key aspects to ensure its success (user, teacher, course, content, design, and technology). In this paper, we have highlighted these aspects besides the implementations of TQM in e-learning by using PDCA Cycle and Six Sigma and challenges that may face it.

\section{REFERENCES}

[1] Arkorful, V. \& Abaidoo, N.(2015).The role of e-learning, advantages and disadvantages of its adoption in higher education. International Journal of Instructional Technology and Distance Learning, 12, 29-42

[2] Best, M. (2005). W Edwards Deming: father of quality management, patient and composer. Quality and Safety in Health Care, 14(4), 310-312

[3] Chatters, B. (2002). Total Quality Management in Software Development. Encyclopedia of Software Engineering.

[4] Jammal, M., Khoja, S., \& Aziz, A. A. (2015). Total Quality Management Revival and Six Sigma. International Journal of Computer Applications, 119(8), 1-5.

[5] Kanji, G. K., Malek, A., \& Tambi, B. A. (1999). Total quality management in UK higher education institutions. Total Quality Management, 10(1), 129-153.

[6] Kaplan, R. S. (1983). Measuring manufacturing performance: a new challenge for managerial accounting research. Readings in Accounting for Management Control, 284-306.

[7] Lakhe, R., \& Mohanty, R. (1994). Total Quality Management. International Journal of Quality \& Reliability Management, 11(9), 9-33.

[8] Little, B.(2003). "Six sigma” techniques improve the quality of e-learning Industrial and Commercial Training, MCB UP Ltd, 35, 104-108

[9] Maruyama, T., \& Inoue, M. (2016). Continuous Quality Improvement of Leadership Education Program Through PDCA Cycle. China-USA Business Review, 15(01).

[10] Oliver, R. (2005). Quality assurance and e-learning: blue skies and pragmatism. Research in Learning Technology, 13(3).

[11] Ozkan, S., \& Koseler, R. (2009). Multi-dimensional students' evaluation of e-learning systems in the higher education context: An empirical investigation. Computers \& Education, 53(4), 1285-1296.

[12] Roffe, I. (2002). E- learning: engagement, enhancement and execution. Quality Assurance in Education, 10(1), 40-50.

[13] Rao, S. S., Ragu-Nathan, T. S., \& Solis, L. E. (1997). Does ISO 9000 have an effect on quality management practices? An international empirical study. Total Quality Management, 8(6), 335-346.

[14] Welsh, E. T., Wanberg, C. R., Brown, K. G., \& Simmering, M. J. (2003). E-learning: emerging uses, empirical results and future directions. International Journal of Training and Development, 7(4), 245-258.

[15] Young, A. F. (2006). Developing Material for Online Management Education - a UK eUniversity Experience. The International Journal of Management Education, 5(1), 45-55. 